\title{
ANALISI DI TRE EFFETTI GALVANOMAGNETICI CONFERNA DI UN NUOVO EFFETTO ").
}

\author{
Memoria del DOTI. A. SELLERIO.
}

Il sottotitolo del presente lavoro, del quale pubblicai un rapido sunto nei $C . R .^{2}$ ), può parer'e un po' arrischiato, perchè sugli effetti galvanomagnetici vi è più di un centinaio di memorie e note che non si possono consultare tutte ${ }^{3}$ ). Ma dalle indagini fatte risulta che il fenomeno qui detto nuovo, non è ancora conosciuto, e anzi i due autori che di sfuggita lo ricercarono ebbero risnltato negativo, come dirò a suo tempo. Si tratta di ciò: mettendo un pezzo di bismuto in un campo magnetico uniforme e facendolo traversare da una corrente normale al campo, si produce una d. d. p. lungo una linea $d i$ forza del campo. Perciò chiamerò nel seguito questo effetto assiale, per distinguerlo da quello Hall o trasversale, (ciò̀ normale al campo).

Leggendo I'articolo citato di Auerbach si può essere tratti in errore, perchè alcuni periodi di p. 454 fanno a prima vista pensare a un effetto assiale. Ivi è detto: « ̀̀ notevole che si « è trovato un effetto Hall anche quando la lastrina invece «d'essere normale è parallela alle linee di forza del campo «magnetico. In questa sitnaziono il fenomeno si produce so" lamente in uno dei due casi possibili, e cioè quando la «corrente primaria è normale al campo».

Cousultando però le memorie originali, si trova che uelle esperienze alle quali allude l'A. la lasirima era parallela alle

1) Vedasi M. La Rosa e A. Sellerio. - Effet galkanomagnétique parallele aus lignes de force et normal au courant. C. R., 170, P. 1447, 1920.

2) Ibidem., fascicolo 25 .

$\left.{ }^{3}\right)$ Le più recenti sono sparse su tutte le riviste correnti; per quelle relativamonte più antiche, cito l'articulo riassuntivo al Auerbach nel volune V, II, parte del Manuale di Winkelmaun, 1905. 
linee di forza e la corrente era normale al campo, ma gli elettrodi secondari erano saldati sulle due facce della lamina, in modo da trovarsi lungo una normale al campo: in quelle esperienze si tratta dunque di un vero e proprio fenomeno Hall, misurato lungo lo spessore della lastrina rettangolare, anzichè lungo la larghezza, come di consueto. Questo non lia che fare col fenomeno in questione.

La prima esperienza grossolana, diretta a constatare l'effetto assiale, fu fatta con un grosso pezzo di bismuto che avevo sottomano, più graude di due scudi d'argento sovrapposti, disposto al solito normalmente al campo prodotto da un'elettrocalamita Weyss e traversato al solito da una corrente normale al campo.

Gli elettrodi secondari erano saldati uno su una faccia e l'altro sull'altra, in modo da trovarsi su una retta normale alla lastra e lungo una linea di forza del campo. In queste condizioni, eccitata l'elettrocalamita, si ebbe una piccola deviazione, primo iudizio dell'effetto assiale ${ }^{1}$ ). In seguito cambiai il pezzo esaminato e andai raffinando la disposizione sperimentale, in modo da allontanarmi alquanto dal punto ci partenza, per seguire lo svolgimento naturale, che l'esperienza stessa suggeriva, come andrò indicando.

Le esperienze promisero fin da principio buon frutto; le misure eseguite misero in luce delle anomalie insospettate, attribuibili - almeno in linea provvisoria - a un altro effetto, che nel seguito sarà chiamato perturbazione. Il metodo che esporrò consente di scindere fra loro $i$ tre effetti (Hall, assiale, perturbazione), o se si vuole le tre manifestazioni di effetti galvanomagnetici.

Supporrò sempre, per comodità il campo magnetico orizzontale, diretto o dall'avanti all'indietro (indicazione $(\cdot)$ ) di

1) Di altre esperienze analoghe, fatte con dischi, di spessore pure considerevole, ma di forma più regolare, a stato già riferito nella nota di M. La Rosa ed A. Sellerio citata. Ivi d anche detto che il fenomeno fu oonstatato anche nel tellurio. 
un osservatore, o viceversa (indicazione $(X)$ ); la corrente primaria diretta o da sinistra a destra (indicazione $\rightarrow$ ), o viceversa (indicazione «). Ho sempre usato elettrodi puntiformi: la retta che unisce quelli primari si dirà asse della lastrina e sarà sempre normale al campo ed orizzontale.

§. 1. - La prima obbiezione che si può fare alle esperienze grezze accenmate è la seguente: la deviazione galvanometrica constatata non è senz'altro prova sufficiente dell'effetto assiale perchè, per poco che uno degli elettrodi secondari sia più alto dell'altro, si ha nn effetto Hall nel senso verticale, che si sovrappone a quello assiale, che, se esiste, ̀̀ orizzontale come il campo. E infatti, girando la lastrina intorno al proprio asse, la deviazione cambiava e si invertiva di segno.

Feci allora alcune misure, mantenendo invariata l'eccitazione e la corr. primaria e girando la lastra, e per ogni posizione notavo le due letture, una col campo $(X)$, l'altra al contrario. Sia qui che in seguito, $p$ denota l'angolo che la congiungente gli attacchi secondari forma con l'asse del campo, ossia con un piano orizzontale.

In questa esperienza, in particolare, $\varphi$ coincide con l'angolo formato dalla lastra e dal piano verticale (parallelo alle facce polari) passante per l'asse della medesima; ed appunto apprezzando (quasi ad occhio) quest' nltimo angolo, che è più visibile, fu valutato $\varphi$. Lastra verticale $e$, per conseguenza, elettrodi secondari in linea orizzontale, corrispondono a $\varphi=0$.

Con campo e corrente primaria costanti, si ottennero le seguenti deviazioni :

\begin{tabular}{c|c|c}
\hline & $\begin{array}{c}\text { (I) campo } \\
\text { in un verso }\end{array}$ & $\begin{array}{c}\text { (II) eampo } \\
\text { opposto }\end{array}$ \\
\hline $\begin{array}{c}0^{\circ} \\
+5^{\circ}\end{array}$ & -4 & +8 \\
$+\mathrm{da} 10^{\circ}$ a $15^{\circ}$ & $+10,5$ & $\pm 7,5$ \\
$+\mathrm{da} 15^{\circ}$ a $20^{\circ}$ & +14 & $\pm 4,5$ \\
$+\mathrm{da} 20^{\circ}$ a $25^{\circ}$ & +22 & -10 \\
$-\mathrm{da} 15^{\circ}$ a $20^{\circ}$ & -21 & $+17,5$ \\
$-\mathrm{da} 20^{\circ}$ a $25^{\circ}$ & -30 & +28 \\
& & +35
\end{tabular}


Bisogna guardarsi da una causa di errore banale che sí ha quando l'elettrocalamita si riscalda, perche allora le saldature secondarie si riscaldano più o meno, secondo la loro vicinanza alle facce polari, dando un notevole effetto termoelettrico, che si riconosce subito, se non altro, per il fatto che spostando anche di poco la lastra in avanti o in dietro la deviazione del galvanometro cambia e di molto (anche se per un momento si interrompe la corrente d'eccitazione). Conoscendo la causa d'errore, è facile sceverarla, o pensare al. rimedio, anche se non si dispone di una elettrocalamita a raffreddamento d'acqua. To ritenni che la cosa più semplice e più sicura fosse di evitare il riscaldamento, chiudendo la corrente di eccitazione solo i pochi minuti, o i pochi secondi necessari per la lettura; così appunto facevo, operavo, ciò, $a$ freddo. Malgrado l' imprecisa valutazione degli angoli, segnando su un diagrammale $\varphi$ come ascisse e le letture come ordinate, si ottengono nettamente due rette, le quali mostrano che l'effetto cercato - ciò̀ quello assiale - $c^{\prime} \grave{e}$, dal momento che per $\varphi=0$ il galvanometro accusa deviazioni $\left.{ }^{1}\right)$.

Ynoltre la figura rivela un fatto strano, e cioè che girando la lastra, si trova una prima posizione (qui $=3^{\circ}$ ) nella quale un effetto si annulla per poi cambiar di segno, mentre l'effetto col campo invertito ha un valore diverso da zero; continuando a girare, si trova nna seconda posizione $\left(=8^{\circ}\right)$ ben distinta, nella quale avviene il contrario. Insomma i dae effetti $(X),(\cdot)$ non si invertono simultaneamente. Tale particolarità del fenomeno fornisce una seconda e più sicura prova dell'effetto assiale, perchè in fondo, se l'origine degli angoli non è esatta le due rette della fig. vanno trasportate insieme o verso destra

1) Se gli elettrodi secondari non sono saldati proprio esattamente su una normale alla lastra, il piccolo erroro si fa sentire molto negli angoli $\varphi$ (valutati guardando la lastra) piccoli, ma è assorbito dagli angoli $\varphi$ grandi; quindi la determinazione della deviazione galvanometrica per $\varphi=0$ fatta indirettamente, ciò̀ vedendo dove le rette della fig. tagliano l'asse delle ascisse, merita filucia. 
o verso sinistra; se così facendo, una delle due viene a passare per l'origine delle coordinate, non potrà passarvi l'altra, quindi si deve concludere che almeno in uno dei casi $(X),(\cdot)$, c'è effetto assiale. Questi risultati del resto vennero poi confermati appieno e lumeggiati dalle esperienze seguenti, con esatta misura di angoli.

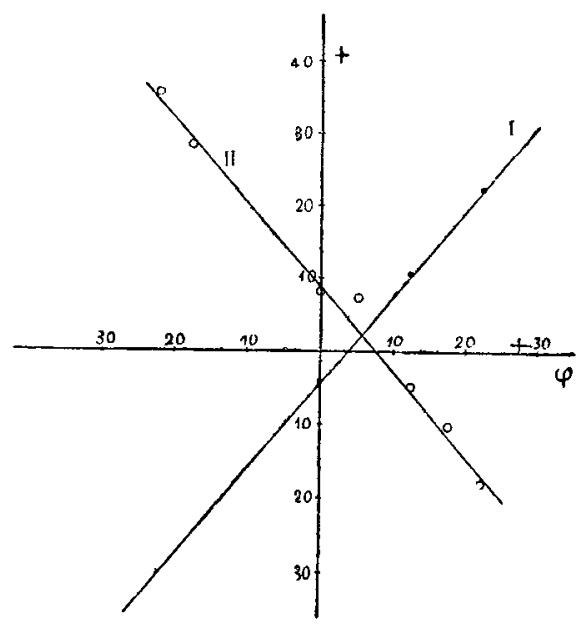

fig 1

Intanto pensai di mettermi nelle condizioni più sempliei, prendendo una lastrina rettangolare molto sottile, secondo il seguente schizzo teorico: Siano A, B gli elettrodi secondari della lastrina vista di profilo, cioè guardata nella direzione del proprio usse, il quale si proietta in $\mathrm{P}$ insieme con gli elettrodi primari.

L'effetto Hall consueto, o trasversale, consiste in una forza elettrica $y$ in direzione verticale, la quale si può decomporre in una $y^{\prime}$ efficace - secondo la retta $A B$, e in un'altra inefficace. Girando quindi la lastrina, si otterrà al galvanometro una deviazione proporzionale a

$$
y^{\prime}=y \operatorname{sen} \varphi
$$


e quindi, se si riportano su un diagramma gli angoli come ascisse e le letture come ordinate, si avrà una sinusoide pas. sante per l'origine delle coordinate. Così l'effetto è nullo per $\varphi==0$, e ivi si ha il punto d'inversione.

Nelie vicinanze del flesso la sinusoide diventa quasi una retta, e queșto giustifica la fig. 1. Se poi il campo si inverte, è come se si aumentasse $\varphi$ di $180^{\circ}$, e quindi si ha lo stesso effetto cambiato di segno, ossia una sinusoide simmetrica rispetto all'asse delle ascisse.

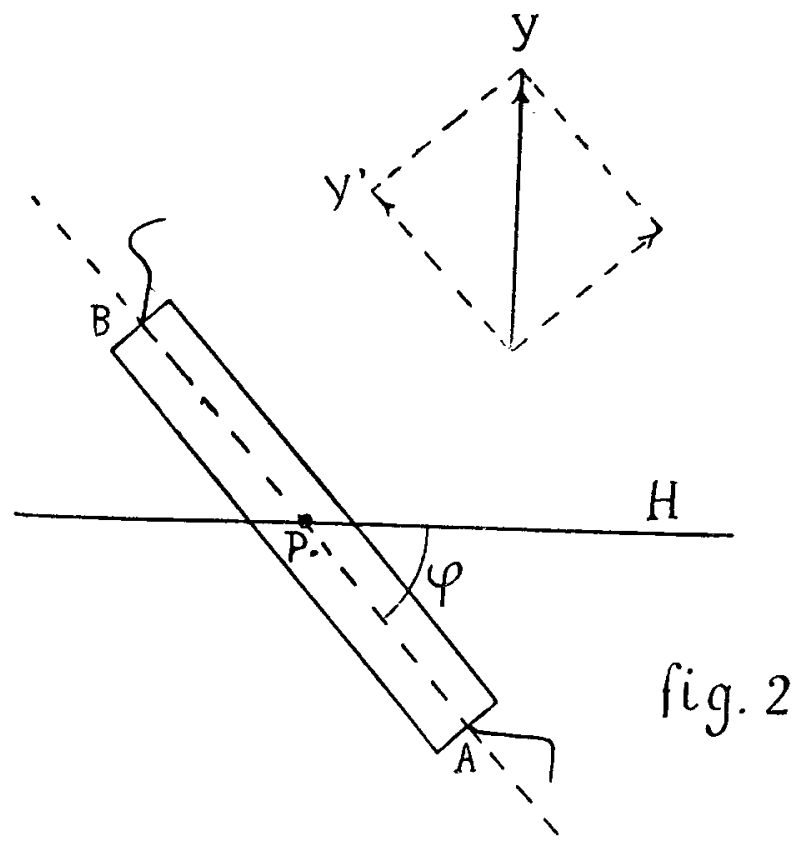

Quando la lastrina è verticale, come nelle disposizioni consuete, l'effetto raggiunge il massimo ed ha per misura secondo. la teoria elettronica

$$
\mathrm{V}_{\mathrm{o}}=\frac{\mathrm{H} i}{\mathrm{~N} e d}
$$

dove, al solito, $\mathrm{N}$ è il numero di elettroni liberi per $\mathrm{cm}^{3}, e$ la. loro carica $d$ lo spessore della lastrina: nelle posizioni incli- 
nate, restando la distanza AB invariata, si avrà evidentemente

$$
V_{0} \operatorname{sen} \varphi
$$

Supponiamo ora che vi sia un effetto nella direzione del campo. In modo perfettamente analogo si avrà una componente efficace

$$
Z^{\prime}=Z \cos \varphi \text {. }
$$

che da il suo massimo effetto $v_{0}$ quando la lastrina è orizzontale, si annulla quando essa e verticale e nelle posizioni intermedie diviene

$$
v_{0} \cos \varphi .
$$

Se i due effetti coesistono, se ne avrà uno risultante

$$
\mathrm{V}=\nabla_{0} \operatorname{sen} \varphi+v_{0} \cos \varphi
$$

che dà ancora una sinusoide

$$
V=w \operatorname{sen}\left(\varphi-\varphi_{0}\right)
$$

sfasata per un angolo $\varphi_{0}$. Il valore 20 misura il massimo effetto risultante e la

$$
\operatorname{tang} \varphi_{0}=\frac{v_{0}}{V_{0}}
$$

il rapporto fra l'effetto assiale e il trasversale. La sinusoide si potrà studiare in vari modi, il più ovvio dei quali è quello di far variare $\varphi$ da $0^{\circ}$ a $360^{\circ}$, mantenendo costante il campo e la corrente primaria. Se non che, molte letture richiedono un certo tempo, durante il quale possono intervenire cause di errori di varia natura non facilmente controllabili, e principali fra tutte - variazioni di temperatura. Un secondo modo, un po' più spiccio, consiste nel girare la lastrina finchè si raggiungono i massimi e i minimi dell'effetto e notare le posizizioni $\varphi_{\max }$. Ma siccome una funzione nelle vicinanze di un massimo ha una variazione molto lenta, $i$ valori di $\varphi_{\max }$ non risulterebbero determinati con precisione, mentre qui ne è necessaria molta, trattandosi di calcolare $\varphi_{0}=\varphi_{\max }-90^{\circ}$, 
che è un piccolo angolo, come è piccolo $v_{0}$ di fronte a $\mathrm{V}_{0}$. Un terzo modo, il più semplice e rapido, consiste nello studiare l'effetto nelle vicinanze della zona neutra, o zona di inversione, sia perchè ivi la funzione ha rapidissima variazione, sia perchè ivi la curva si riduce a una retta - come ho trovato pienamente confermato dall'esperienza - e bastano

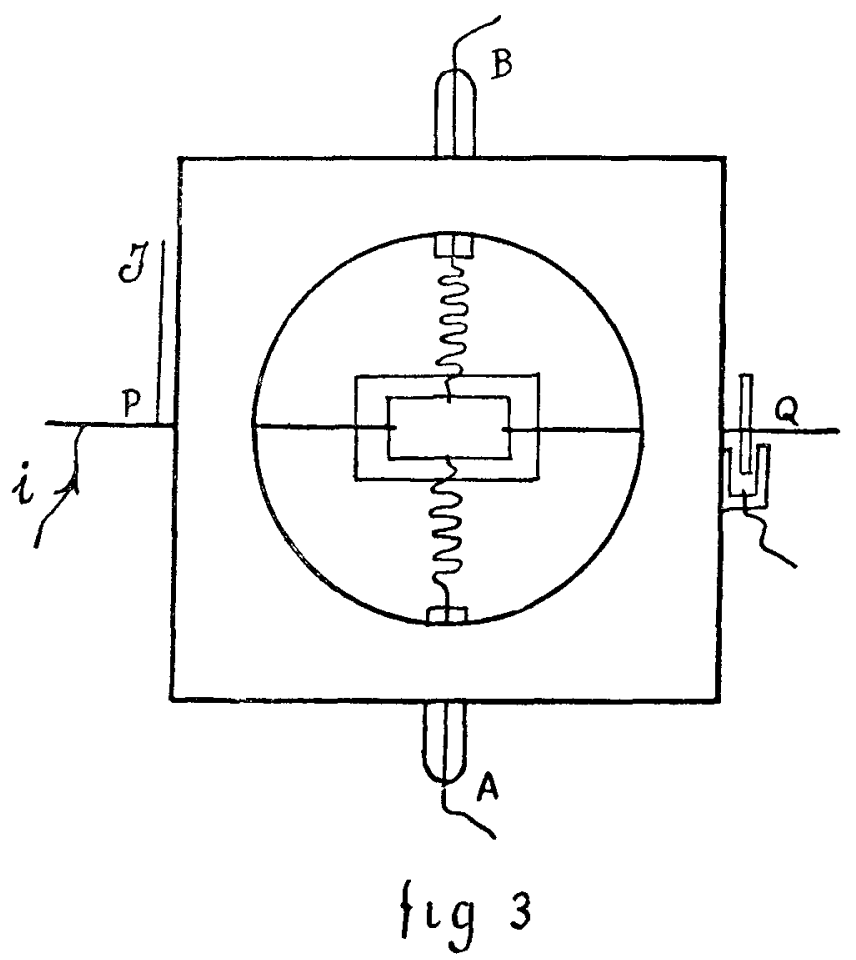

due punti ben determinati per individuarla. Secondo questo ultimo criterio, costruii un piccolo apparecchio disegnato schematicamente in figura. Una lastrina di mica può girare intorno all'asse metallico $\mathrm{PQ}$ dentro un telaino di legno; l'asse porta dalla parte di $\mathrm{P}$ un indice $\mathrm{I}$ che insiste su un settore guaduato. Sulla mica è adattata la lastrina di bismuto da sperimentare, connessa mediante l'asse al cicuito primario e 
mediante fili flessibilissimi verticali al circuito del galvanometro. In $\mathbf{A}$ e B, tubicini di vetro per assicurare l' isolamento.

Gli attacchi secondari erano fissati in principio con piccoli morsetti di rame che dovetti tosto abbandonare, sia perchè non davano un contatto sicuro perfettamente immutabile durante la rotazione della lastrina, sia perchè erano un vero magazzino di calore e dovetti usare quindi le saldature, che avevo prima cercato di evitare, per lo scrupolo di non introdurre altre forze termoelettriche, oltre quelle certamenie esistenti fra rame e bismuto. Un'altra imperfezione può essere causa di errori e cioè: se in $\mathbf{P}$ e in $Q$, i fili che portano la corrente primaria sono saldati o stretti a vite, essi, per quanto teneri siano, quando si gira la lastrina possono formare uno con l'altro una coppia che distorce la mica, e ne risente anche la lastrina. Questo è un grave inconveniente '), e, per toglierlo del tutto, stabilii in $Q$ un contatto a mercurio mediante un pozzetto di sughero e una rotellina saldata all'asse.

Con queste ed altre lievi accortezze, dirette in gran parte ad evitar disturbi termici, le esperienze vennero di una grande nettezza e precisione. La corrente primaria era fornita dia accumulatori, quella d'eccitazione era presa sulla rete stradale, o più spesso, data l'incostanza della tensione, era fornita pure da accumulatori. Non ho usato alcun metodo di compensazione per non complicare, ma invece, mediante ripetuti tentativi (spostando un poco le saldature, o raschiando lievemente l'orlo della lastrina) ho ottenuto che gli attacchi secondari a campo nullo fossero quasi esattamente su una linea equipotenziale, in modo che il galvanometro desse una deviazione trascurabile. Quando poi era apprezzabile, l'effetto veniva misurato dalla

1) È noto che si cercò di spiegare addirittura l'effetto Hall ridncendolo a deformazioni meccaniche, che le lastrine subirebbero nel campo magnetico, e appunto il fenomeno si può imitare mediante azioni meccaniche e termiche esercitate stlla lastrina. Vedasi S. Bidwell Phil. Mag (5), volume 17 , p. 250,1884 , o anche l'articolo riassuntivo sugli effetti galvanomagnetici nel vol. LI, $1895 \mathrm{del}$ Wiedemann. 
differenza fra le letture con e senza campo. Le misure deI campo furono fatte con una spiralina di Leduc. Le facce polari avevano il diametro di $7 \mathrm{~cm}$. In quanto all' uniformità ho da ritenerla completa nella regione utilizzata, perchè gli effetti non venivano sensibilmente alterati, spostando la spiralina di Leduc o lastrine esaminate, parallelamente a sè stesse dentro il campo, verso destra sinistra, alto basso, avanti e dietro almeno di uno, o due centimetri.

La laminetta di bismuto provata più a lungo aveva piccolo formato $(16 \mathrm{~mm} . \times 11 \mathrm{~mm}$.) e uno spessore medio di 0,25 millim. Quando non sia espressamente avvertito il contrario, si sottintendono le seguenti condizioni:

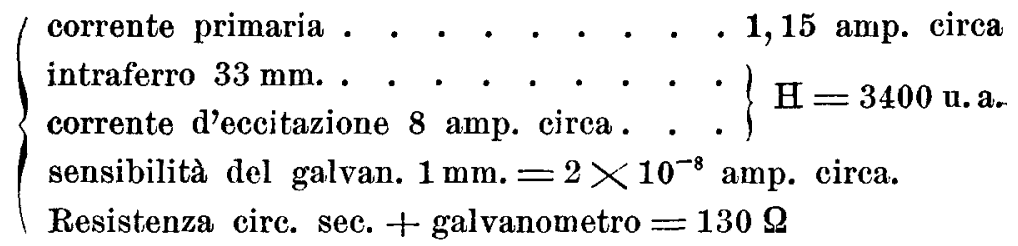
cosicchè $1 \mathrm{~mm} .=2,6$ microvolt circa.

Si ha la tabellina:

\begin{tabular}{c|c|c|c|c}
\hline & $5^{0}$ & $10^{\circ}$ & $15^{\circ}$ & $20^{\circ}$ \\
\hline$(\cdot)$ & +17 & +2 & $-8,5$ & -23 \\
$+(\cdot)$ & $-16,5$ & -3 & +10 & +22 \\
$+(X)$ & -36 & -22 & -10 & +4 \\
+36 & +25 & +10 & -3
\end{tabular}

Anche qui, come con la lastra grossa di bismuto, si constata l'esistenza di un effétto per $\varphi=0$ che corrisponde ad nua componente lungo il campo, cioè si ha l'effetto che chiamai assiale. Appare inoltre manifesto che:

1. - Invertendo la corrente primaria, l'effetto si inverte simmetricamente, e le due curve si tagliano in un punto dello 
asse delle ascisse. Se poi si fa variare la corrente primaria, si constata facilmente che l'effetto varia proporzionalmente come avviene in tutti i fenomeni galvano e termomagnetici ').

Pertanto, per uno stesso campo e correnti diverse, si ha un fascio di curve che si incrociano sull'asse delle $\varphi$.

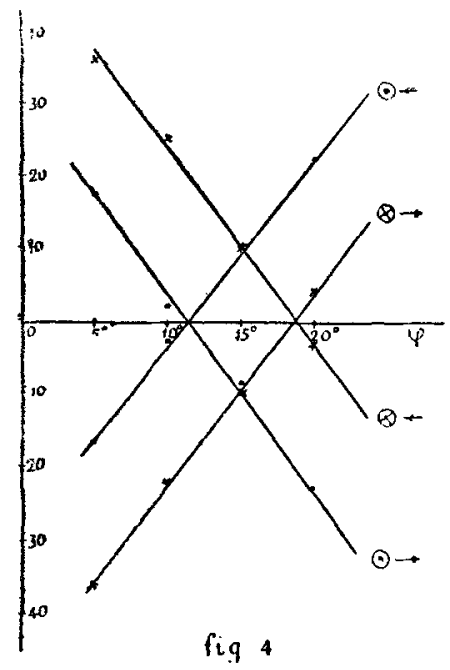

2. - Invertendo solo il campo, l'effetto non si inverte simmetricamente. E questa la solita dissimetria osservata bene per il primo da Righi ") nello studio dell'effetto Hall comune $\left(\varphi=90^{\circ}\right)$. Qui siamo penetrati, per così dire, nel cuore di tale dissimetria, come apparirà dal seguito.

3. - Girando la lastrina si trova una prima posiziona $\varphi_{1}$, (nel caso presente circa $11^{\circ}, 5$ ) nella quale l'effetto $(\cdot)$ si annulla per poi cambiar segno, menrre l'effetto $(X)$ ha un valore diverso da zero; e si trova poi una seconda posizione $\varphi_{3}$ (nel caso

1) Sembrano fare eccezioni le correnti debolissime, come sospetto gia Righi - Nuovo Cimento (3.a), XV, 1884, pag. 130 - e conferǹò poi Moretto Nuovo Cimento $\left(4 .^{\mathrm{a}}\right) 11,1900$, pag. p. 286.

$\left.{ }^{2}\right)$ l. c., pag. 133 . 
presente $19^{\circ}$ circa) ben distinta dalla prima, nella quale avviene il contrario.

Questo fatto gia intravisto col grosso pezzo di bismuto, fig. 1, è accertato ora con sicurezza; esso appare strano perchè, malgrado la dissimmetria dei valori, si poteva pensare che i due effetti $(\cdot),(X)$ si annullassero sempre simultaneamente o annullando la corrente, o annullando il campo, o girando la lastrina, in modo che raggiungesse una certa posizione. Invece le posizioni di inversione sono due distinte.

4. - Due curve $(\cdot),(X)$ relative ad una medesima corrente primaria si incontrano in un punto ( $q$ ui avviene per $\varphi=15^{\circ}$ circa); ciò significa che vi è upa posizione della lastrina nella quale l'effetto non cambia nè di valore, nè di segno mutando il senso del campo, come si verifica direttamente con l'esperienza. Anche quasta è una circostanza insospettata. Nel caso attuale, questo effetto $y_{0}$ è circa 10 , e non è imputabile a cause di errore, perchè cambiando la corrente d'eccitazione esso varia - come si vedrà - molto rapidamcnte. Se la lastrina si lasciasse in tale posizione in un campo alternato, liberandosi dagli effetti di induzione, si avrebbe agli elettrodi secondari una d. d. p. di segno costante. La direzione $\varphi_{0}$, che è la bisettrice dell'angolo formato dalle direzioni $\varphi_{1}, \varphi_{2}$ si chiamerà asse di inversione.

5. - In vicinanza dell'asse di inversione, le quattro curve $(\cdot) \leftrightarrow,(X) \leftrightarrow,(\cdot) *,(X) \ll$ si riducono sensibilmente a quattro rette di uguale pendenza ( \pm ) cosicchè a due a due sono parallele, ossia: le letture fatte invertendo campo e corrente differiscono per una grandezza costante $=2 y_{0}$. Se essa si annullasse, le quattro rette si trasporterebbero due un po' più su, e due un po' più giù per il segmento $y_{0}$, verrebbero a tagliarsi sull'asse delle $\varphi$, e a due a due coinciderebbero: il fenomeno riprenderebbe la simmetria anche rispetto al campo.

Anche col grosso pezzo di bismuto ci trovavamo (fig. 1) nelle stesse condizioni, il che si riconosce osservando che la somma algebrica delle letture fatte cambiando solo il senso 
del campo mantiene un valore presso che costante quando $\varphi$ varia.

Ora questo $y_{0}$, causa - o meglio manifestazione - della dissimmetria, il quale varia e rapidamente col campo, ma non dipende dal verso corrisponde evidentemente ad un effetto che si può pensare sovrapposto al principale; il più semplice, considerando che deve essere funzione pari del campo, è di supporlo proporzionale ad $\mathrm{H}^{x}$. E variando l'eccitazione si trovò appunto che $y_{0}$ si raddoppia quando il campo cresce in un rapporto non molto diverso da $1: \sqrt{2}$ civè da 3400 a 5300 Gauss, come si può rilevare costru zudo il diagramma per la seguente tabellina:

\begin{tabular}{|c|c|c|}
\hline & $\hat{\mathrm{c}}=0^{\circ}$ & $\varphi=30^{\circ}$ \\
\hline$(\cdot) \ggg$ & +37 & -66 \\
\hline$(\cdot)$ & -40 & +65 \\
\hline$(X) \ggg$ & -79 & +35 \\
\hline$(x) \ll$ & +77 & -35 \\
\hline
\end{tabular}

Invece l'inclinazione delle quattro rette, che va di pari passo col fenomeno principale, risulta maggiore allo incirca nella stessa proporzione in cui è cresciuto il campo.

Le prove furono poi replicate facendo variare molto il campo, e prendendo per ogni retta due punti: si ebbe così la fig. 5 .

Dopo ciò, le quattro rette si possono mettere subito in equazione. Considerandone una qualnnque, e tenendo conto che passa per il punto $\left(\vartheta_{0}, y_{0}\right)$ la sua equazione si scrive:

$$
y-y_{0}=\left(\varphi-\varphi_{0}\right) k \text {. }
$$

In essa, $k$ che dà l'inclinazione cambia di segno col campo $\mathrm{H}$, e gli si mantiene sensibilmente proporzionale; $y_{0}$ non 
cambia di segno con $H$ ed è in prima approssimazione proporzionale ad $\mathrm{H}^{2}$; l' uno e l'altro sono poi proporzionali alla corrente primaria. Invece $\varphi_{0}$ è quasi invariabile, quindi l'equazione denotando con $\mathrm{A}, \mathrm{C}$ delle costanti diventa

$$
y=\mathrm{A} H i\left(\varphi-\varphi_{0}\right)-\mathrm{CH}^{2} i
$$

e comprende tutte le quattro rette, Questa equazione rappresenta quindi con buona approssimazione tutto il fenomeno e cioè tutti i quattro casi $(\cdot) \leftrightarrow,(\cdot)$ vicinanze dell'asse di inversione. L'approssimazione è tanto migliore quanto più debole è il campo.

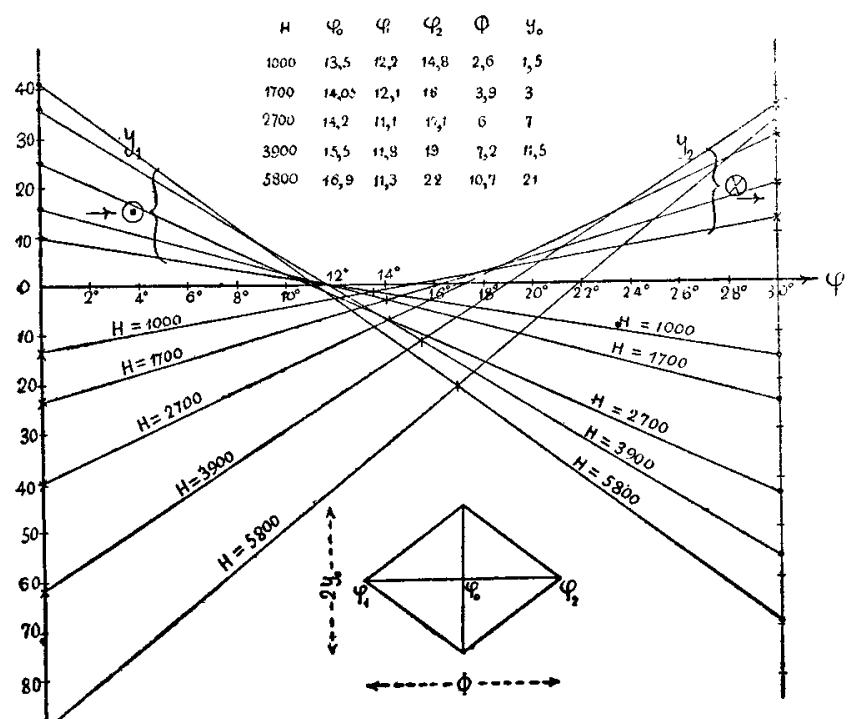

figs

Se nella (4), e nella (3) da cui essa discende, non vi fosse il termine in $\mathrm{H}^{2}$, l'esperienza risponderebbe bene alle previsioni teoriche contenutè nella (2), giacchè per angoli $\varphi-\varphi_{0}$ non molto piccoli l'equazione

$$
y=k\left(\varphi-\varphi_{0}\right)
$$


coincide con

$$
y=w \operatorname{sen}\left(\varphi-\varphi_{0}\right)
$$

Dunque si deve concludere che da un canto vi sono i fenomeni previsti, dall'altro ve ne è uno imprevisto che vi si sovrappone. Si hanno così:

1. - Un effetto Hall trasversale al campo.

2. - Un altro effetto assiale Iungo il campo.

3. - Un effetto dipendente da $\mathrm{H}^{2}$, che sarà chiamato perturbazione.

§. 2. - Ammesso l'effetto perturbatore, è ragionevole anche ammettere che esso agisca non solo nelle vicinanze dell'asse di inversione, ma anche in ogni posizione della lastrina, e quindi che si debba correggere la (2) con laggiunta di un termine in $\mathrm{H}^{2} i$. Se questo termine è costante - ipotesi $a$ ) le sinusoidi sono spostate come le rette della fig. 7 in su 0 in giù, secondo un'equazione

$$
y=k \operatorname{sen}\left(\varphi-\varphi_{0}\right)-\mathbf{O H}^{2} i
$$

Ma si può pure pensare - ipotesi $b$ ) - che esse siano spostate verso destra o sinistra secondo un'equazione

$$
y=\mathbf{A} \mathbf{H} i \operatorname{sen}\left(\varphi-\varphi_{0}+a \mathbf{H}\right)
$$

giacchè anch'essa per piccoli valori di $\left(\varphi-\varphi_{0}\right)$ e di $a H$ si riduce alla (4). Evidentemente per decidere la cosa non basta studiare il fenomeno nelle vicinanze della zona di inversione ma si deve seguirlo tutto.

Tenni per non cambiar nulla, lo stesso apparecchio, quantanque non fosse adatto per grandi rotazioni, e aggiunto solo un indice ausiliario per rendere possibile la lettura di grandi angoli, procedetti quindi alle misure con variazioni grandi di $\varphi$ nelle identiche condizioni di prima. 
Ed ecco i diagrammi ottenuti

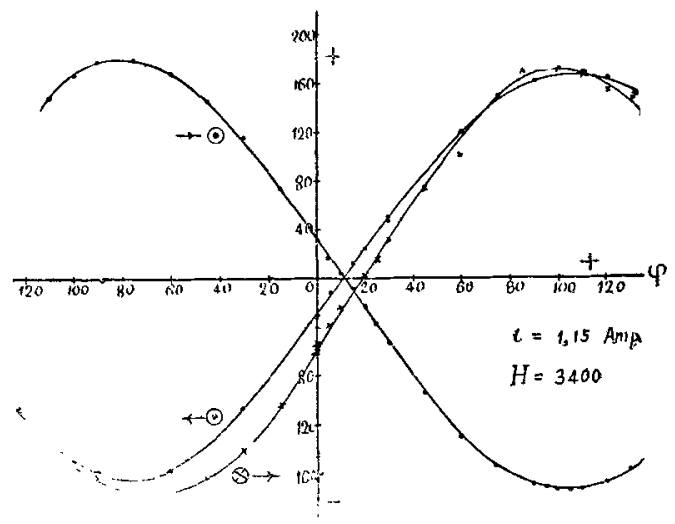

Il risultato ni sembrò però poco chiaro, perchè le curve si intrecciano ed ebbi l'idea naturale di attribuire questo fatto ad errori di osservazione, giacchè nel tempo occorrente per prendere 50, o 60 letture possono mutare vari fattori, e quindi si può ammettere che le misure non siano ben coerenti.

Per analizzare il fenomeno con sicurezza non rimane al. lora che lo studio dei massimi e dei minimi già accennato, perchè nella ipotesi a) le curve si presenteranno come nella fig. 7 a sinistra coi massimi di valore diverso e nello stesso posto; nell'altra ipotesi, i massimi avranno lo stesso valore ma saranno in posti diversi e lo spostamento dei massimi sarà eguale a quello dei punti nentri. L'inconveniente già cenuato della poca precisione nel determinare i massimi (o minimi) si gira, prendendo alcuni punti nelle loro vicinanze e le difficoltà si dividono determinando, separatamente prima dove i massimi si trovano e poi quali valori hanno.

Cosi ho accertato:

1. - I valori assolnti dei massimi sono quasi eguali (nek caso precedente circa 175 ) per tutte le quattro curre ').

1) La curva $(\cdot) \ggg$ della fig. 7 e effettiramente un po' errata in basso, ma è giu ta nel resto. 
2. - Le loro posizioni cambiano, cambiando il verso del campo, ma restano immutate cambiando il verso della corrente primaria.

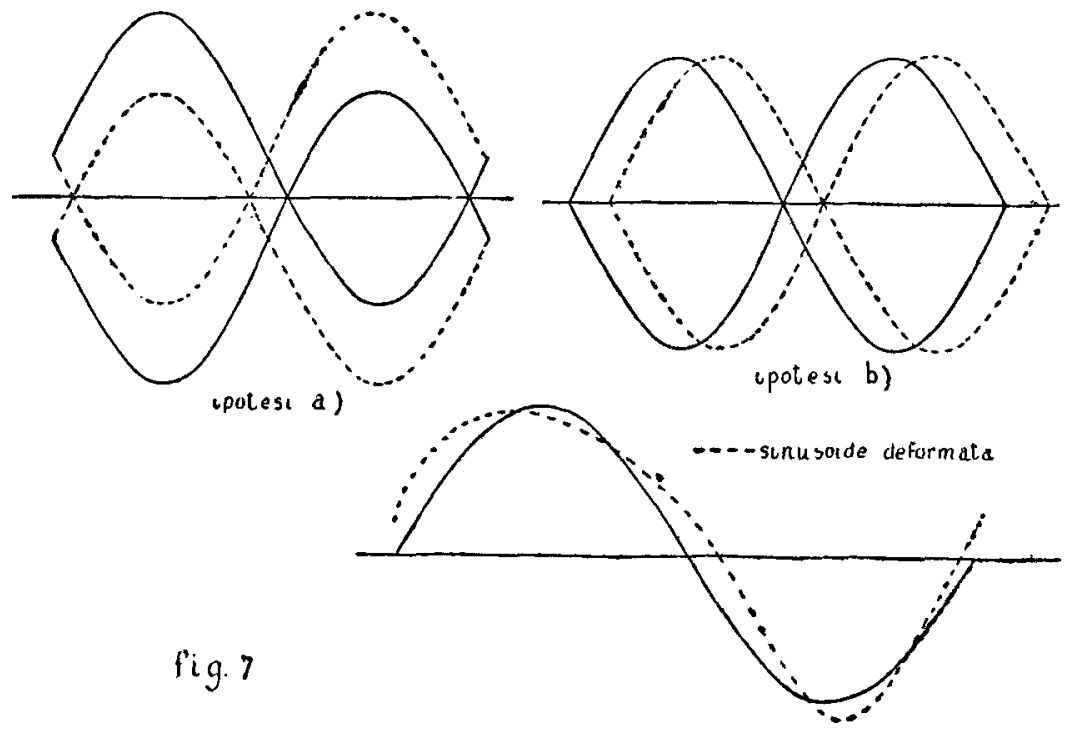

Nel caso presente queste posizioni sono:

$$
\begin{aligned}
& \text { per }(\cdot)+106^{\circ}-80^{\circ} \\
& \text { per }(X)+105^{\circ}-72^{\circ}
\end{aligned}
$$

Questi dati non vanno d'accordo completamente nè con l'ipotesi $a$ ), nè con l'ipotesi $b$ ) espresse nella fig. 7. Fissiamo l'attenzione su una coppia di curve vicine (per es. $(\cdot) \ll(X) \rightarrow$ ): queste hanno quasi gli stessi valori dei massimi, e quindi non si possono dire spostate in su, una rispetto all'altra, ma neanche si può pensare ad uno spostamento verso destra (ipotesi $b)$, perchè mentre i punti neutri, secondo la fig. 4 distano $\varphi_{2}-\varphi_{1}=19-11,5=\overline{\mathbf{7}}, 5$ gradi, $\mathrm{i}$ massimi positivi distano 106-105 = 1 grado, e i massimi negativi di 80-72=8 gradi. Quindi le due curve debbono intrecciarsi proprio, come avviene nella fig. 6, sospettata erronea. 
Insomma deve trattarsi di sinusoidi deformate, tanto è vero che la distanza fra il massimo e il minimo è

$$
\begin{aligned}
& 106+80=186^{\circ} \text { per }(\cdot) \\
& 105+71=176^{\circ} \text { per }(X)
\end{aligned}
$$

invece di 180, cosicchè la prima sinusoide è slargata, la seconda è contratta. Confroutando (fig. 7) una di queste sinusoidi così deformate (esagerando) con una ideale, si trova che esse differiscono per una grandezza che si annulla in ogni punto di incontro e cambia seguo dalle due parti di esso. Poichè nel corso di un giro, tutto si deve ripetere periodicamente, si può completare il risultato come in figura - oltrepassando alquanto per estrapolazione i dati sperimentali che vanno $\mathrm{da}+130^{\circ} \mathrm{a}-120^{\circ}$, con un intervallo di $250^{\circ}$ gradi.

Cosi si rivela questo fatto strano che il fenomeno detto perturbazione, il quale deforma le curve facendole sensibil: mente abbassare, o innalzare nelle vicinanze dell'asse di inversione, cambia quattro volte di segno nel corso di un giro; si manifesta cioè come un'armonica del fenomeno principale. Se esso non vi fosse, le quattro curve $(\cdot) \rightsquigarrow,(\cdot) *(X) \cdots,(X)$ sarebbero esattamente sinusoidi e si incontrerebbero in un unico punto sull'asse delle $\varphi$ anzichè nei quattro vertici di un quadrilatore, come nella fig. 4. Questo quadrilatero si può dire il nocciolo di tutto il complesso dei fenomeni osservati, giacohè (fig. 5) la diagonale principale $2 y_{0}$ avendo la lunghezza $2 \mathrm{CH}^{2} i$ misura il quadrato del campo e con esso la pertubazione; l'inclinazione $k=w=\mathrm{BH} i$ dei lati misura l'effetto principale che è proporzionale al campo, o risulta alla sua volta composto di uno più cospicuo trasversale e uno assiale. Questi due effetti si possono scindere conoscendo il valore di $\varphi_{0}$ ciò̀ il centro del quadrilatero. L'altra diagonale poi $\Phi=\varphi_{2}-\varphi_{1}$ che chiamerò secondaria, ha una lunghezza indipendente dalla corrente primaria, giacehè secondo quanto 
si disse al $\S 1$ gli estremi si mantengono fissi variando la corrente primaria.

Questa diagonale come si ricava dalla (5) ha la lunghezza

$$
2 y_{0}=2 \frac{\mathrm{C}}{\mathrm{A}} \mathbf{H}
$$

e quindi misura il rapporto fra la perturbazione e il fenomeno principale, nelle vicinanze dell'asse di inversione.

Tornando all'armonica del fenomeno principale, essa ricorda lontanamente le analisi che si fanno in elettrotecnica Alei campi magnetici all' interuo delle macchine. Qui però è da escludersi che possa trattarsi di non perfetta uniformita del campo, perchè - come si disse - spostando parallelamente la lastra nel campo l'effetto non cambia. Piuttosto si può pensare a variazioni di resistenza; ma di ciò mi occuperò in seguito. Prima però di indagare la natura del fenomeño, è prudente accertarlo meglio, mettendolo al coperto da obbiezioni. Si consideri sempre la coppia di curve $(\cdot) \rightarrow,(X)$ e si fissi una posizione $\varphi$ della lastrina. Fatta una prima lettura, per esempio nelle condizioni $(\cdot)$, si invertano campo e corrente primaria e si faccia una seconda lettura. Tutto ciò richiede appena un minuto: se vi sono (lievi) cause di errore, come variazioni nella corrente primaria, o nell'eccitazione, o nella temperatura ambiente, ecc. le due letture saranno sbagliate, ma la loro differenza $\Delta$ sarà presumibilmente esatta. Così ha proceduto per varie posizioni. Vediamo che cosa rappresenta $\Delta$; nell' un caso per esempio $(\cdot) \rightarrow$ la sinusoide pura vien deformata per l'aggiunta di un termine proporzionale ad $\mathrm{H}^{2} \cdot i$, nell'altro caso $(X)$ vien deformata per sottrazione dello stesso termine, giacchè $(-\mathbf{H})^{2}(-i)=-\mathbf{H}^{2} i$, quindi la differenza $\Delta$ delle due letture è uguale al doppio della perturbazione. 
Le misure sono raccolte nella seguerte tabellina con la relativa figura

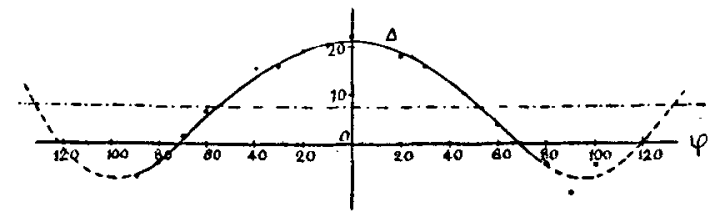

fig 8

\begin{tabular}{r|r|c|c|c|c|c|c|c}
\hline $10^{\circ}$ & $20^{\circ}$ & $30^{\circ}$ & $60^{\circ}$ & $70^{\circ}$ & $80^{\circ}$ & $90^{\circ}$ & $100^{\circ}$ \\
\hline$(\cdot)$ & -5 & +22 & +46 & +112 & +130 & +140 & +149 & +159 \\
$(X) \%$ & -23 & +4 & +30 & +108 & +130 & +144 & +159 & +163 \\
\hline differenza $\Delta$ & +18 & +18 & +16 & +4 & 0 & -4 & -10 & -4
\end{tabular}

\begin{tabular}{r|c|c|c|c|c|c|c|c|c|c|}
\hline & 0 & $-10^{\circ}$ & $-20^{\circ}$ & $-30^{\circ}$ & $-40^{\circ}$ & $-60^{\circ}$ & $-70^{\circ}$ & $-80^{\circ}$ & $-90^{\circ}$ & $-100^{\circ}$ \\
\hline$(\cdot)+$ & -31 & -61 & -84 & -111 & -127 & -157 & -163 & -166 & -159 & -151 \\
$(X)$ & -53 & -82 & -103 & -127 & -143 & -164 & -165 & -162 & -152 & -141 \\
\hline $\operatorname{differenza} \Delta$ & +22 & +21 & +19 & +16 & +16 & +7 & +2 & -4 & -7 & -10
\end{tabular}

E manifesta la regolarita di A come una vera armonica a periodo $\pi$ avente il massimo quasi nella posizione orizzontale della lastrina. Dove $\Delta=0$, le curve $(\cdot),(X) \rightarrow$ si incontrano, cioè invertendo campo e corrente primaria, il galvanometro dà la stessa deviazione: questo avviene, secondo la fig. 8 quattro volte nel corso di un giro, cioè all'ingrosso a $\pm 70^{\circ}, \pm 115^{\circ}$ in discreta concordanza con la fig. 5. Questi quattro punti d'incontro, poichè in essi si annulla la perturbazione sono $\mathrm{i}$ soli punti della sinusoide ideale accessibili direttamente all'esperienza. 
Guardando la figura 8, si può scrivere subito

$$
\Delta=2(5+6 \cos 2 \varphi)
$$

e poichè vi deve essere la proporzionalità col quadrato del campo, e con la corrente primaria, si può ammettere che $\Delta$ abbia verosimilmente la forma

$$
\frac{\Delta}{2}=(a+b \cos 2 \varphi) H^{2} i
$$

Così l'effetto complessivo, con molta approssimazione, verrebbe rappresentato dall'equazione generale

$$
\begin{aligned}
& y=\mathrm{A} \operatorname{sen}\left(\varphi-\varphi_{0}\right) \cdot \mathrm{H} i+(a+b \cos 2 \varphi) \cdot \mathrm{H}^{2} i \\
& =\mathrm{A}^{\prime} \cdot \mathrm{H} i \operatorname{sen} \varphi-\mathrm{B}^{\prime} \cdot \mathrm{H} i \cos \varphi+(a+b \cos 2 \varphi) \mathrm{H}^{2} i \\
& \text { trasversale assiale perturbazione. }
\end{aligned}
$$

Come si vede, il fenomeno è molto complesso, e di gran lunga non basta la solita costante $R$ dell'effetto Hall a rappresentarlo, mentre invece appena sono sufficienti le quattro costanti $\mathrm{A}, \varphi_{0}, a, b$, oppure le equivalenti $\mathbf{A}^{\prime}, \mathrm{B}^{\prime}, a, b$. Intanto la formola stabilita rende conto di tutte le particolarità osservate sperimentalmente e già ricordate, e di altre ancorạ, confermate poi. Fra l'altro voglio notare:

1. - Nelle vicinanze della posizione $d i$ inversione si può porre sensibilmente $\cos \varphi=$ cost $=1$, tanto più che $\mathrm{i}$ coefficenti $a, b$, sono molto piccoli, e quindi si ricade nell'equazione (4), ossia nella fig. 4.

2. - Invertendo campo e corrente primaria, il primo termine della (6) non si altera, e il secondo cambia segno, quindi affinchè i due valori coincidano, occorre che tale secondo termine si annulli: in altre parole se le due curve si incontrano, questo avviene nelle quattro posizioni $\varphi$ che corrispondono alle radici dell'equazione

$$
(a+b \cos 2 p) \mathbf{H}^{\mathbf{y}} i=0 .
$$

Queste posizioni devono essere quindi indipendenti dal campo e dalla corrente. Approssimativamente si trova ap- 
punto così: la verifica esatta di questa proposizione è difficile perchè le curve si incontrano (fig. 6 ) sotto un angolo molto acuto e quindi per un buon tratto le letture relative all'una o all'altra quasi coincidono.

3. - Per $\varphi=90^{\circ}$, che corrisponde alle comuni esperienze sull'effetto Hall, la (7) si riduce semplicemente a:

$$
y=\mathrm{A}^{\prime} \mathrm{H} i+(a-b) \mathrm{H}^{2} i
$$

che contiene 3 costanti.

Siccome $a, b$, sono piccoli, anche la differenza $(a-b)$ è piccola, ma tuttavia non trascurabile, giacchè appunto essa produce la nota dissimetria dei valori rispetto ad $\mathbf{H}$.

Comunemente in vista di questa dissimetria, per determinare la costante $R$, si fanuo due misure col campo in uno $e$ nell'altro senso e poi si prende la media. Questo procedimento è legittimo, perchè cambiando $H$ in $-H$ si ha

$$
\bar{y}=-\mathrm{A}^{\prime} \mathrm{H} i+(a-b) \mathrm{H}^{2} i,
$$

e facendo la media fra $i$ valori numerici che sono

$$
\mathbf{A}^{\prime} \mathrm{H} i+(a-b)^{\prime} \mathrm{H}^{\mathbf{2}} i
$$

nell' un caso e

$$
\mathrm{A}^{\prime} \mathrm{H} i-(a-b) \mathrm{H}^{2} i
$$

nell'altro, si ba appunto solamente $\mathbf{A}^{\prime} \mathrm{H} i$, e restano così eliminati sia l'effetto assiale che l'effetto perturbatore.

Così ridotto, il fenomeno richiede una sola costanre $A^{\prime}$ che equivale ad $\mathbf{R}$.

4. - Il massimo effetto non si ottiene quando la lastrina si mette al solito verticale, ma invece quando la lastrina è girata, o un po' avanti o un po' indietro, e ciò sia a causa dell'effetto assiale che della perturbazione. Quest' ultima anzi fa sì che questo massimo effetto si sposti leggermente al variare di $\mathrm{H}$ come si vede eguagliande a zero la derivata della (6).

5. - Per un campo molto debole, il termine in $\mathbf{H}^{\mathbf{2}}$ che ha un piccolo coefficiente diventa trascurabile del tutto e rimangono solo l'effetto trasversale solito e quello assiale. Il qua- 
drilatero caratteristico della fig. 4 si restringe quasi a un punto, al suo centro. Allora l'effetto è simmetrico rispetto al campo e proporzionale ad esso. A misura che il campo cresce, aumentano le dimensioni del quadrilatero e aumenta la dissimmetria del fenomeno.

La diagonale secondaria $\Phi=\varphi_{2}-\varphi_{1}$ rappresenta l'intervallo dentro il quale l'effetto non cambia segno, invertendo il campo. Questo intervallo, per campi deboli, è ristretto alle vicinanze dell'asse di inversione, cioè quando la lastra è vicina alla posizione orizzontale (a quasi $15^{\circ}$ nel caso nostro), ed ingrandisce a misura che cresce il campo. Se questo cresce molto, può darsi che il termine quadratico in $\mathrm{H}$ finisca del tutto col prevalere sull'altro, nel qual caso in nessuna posizione l'effetto s'invertirebbe cambiando il senso del campo.

Io non sono riuscito a ciò con la lastrina in discorso, $\mathrm{mi}$ sarebbe bisognato un campo intensissimo (forse infinito?) e questo non potevo ottenere sia per la limitata potenzialita dell'elettrocalamita, sia perchè la lastrina girevole non mi permetteva di avvicinare a più dne centimetri e mezzo le facce polari, ma vi sono, come è noto, dei casi nei quali anche per $\varphi=90^{\circ}$, ciò̀ con la laminetta verticale l'effetto nou muta segno invertendo il campo ").

L'equazione dell'effetto per la lastrina esaminata, è approssimativamente

$$
y=160 \operatorname{sen}(\varphi-15)+(5+6 \cos 2 \varphi)
$$

che, avendosi

$$
i=1,15 \text { Amp. } \mathrm{H}=3,4 u . a \times 10^{3},
$$

si può scrivere:

$$
y=41 \mathrm{H} i \operatorname{sen}(p-15)+(0,37+0,44 \cos 2 \varphi) \mathrm{H}^{2} i
$$

Qui l'effetto assiale è all' incirca la quarta parte di quello trasversale perchè

$$
\operatorname{tang} \varphi_{0}=\operatorname{tang} 15=0,27 .
$$

l) Vedi per esempio Righi, l. c., pag. 133. 
Stabilita così in vario modo l'esistenza dell'effetto assiale, ricercai con maggiore diligenza nella letteratura e trovai in proposito due tentativi con esito sfavorevole. Il primo è di Hall stesso "), il quale mise la lastrina nella posizione che ho indicato con $\varphi=0$, e rimase incerto se vi fosse, o no effetto; ma egli operava con foglioline di oro, che dànno risultati di gran lunga inferriori al bismuto. Il secondo tentativo fu fatto da Ettingshausen o Nernst ${ }^{3}$ ) con una laminetta di bismuto: il risultato negativo non sorprende troppo, perchè il bismuto si presenta sotto qualità e aspetti differenti e hanno buon gioco nei fenomeni le proprietà cristalline. Ad ogni modo, quei due tentativi furono fatti di sfuggita e più che altro per scrupolo, giacchè, come è noto, il punto di partenza delle esperienze di Hall fu quello di stabilire se le azioni elettrodinamiche si esercitino contro il conduttore, o contro le linee di corrente e quindi Hall, come tutti in seguito ricercarono azioni in un piano perpendicolare al campo; $\theta$ messo da parte quello scrupolo, nessuno naturalmente si occupò più della cosa.

Con le presenti esperienze, mi pare che l'effetto assiale sia da mettersi fuori dubbio.

§ 3. - Dal punto di vista qualitativo, l'analisi fatta è completa e risolve il fenomeno nei suoi tre elementi: cambiando la lastrina è è da aspettarsi che tutti e tre si presentino in generale, pur ammettendo che nei singoli casi uno, o due possano essere piccolissimi, o annullarsi del tutto.

In quanto alla formola (6), ovvero (7) faccio per altro qualche riserva circa il termine in $\mathrm{H}^{2}$ (piccolo e difficile a determinarsi esattamente), giacchè ripetendo l'esperienza con un campo più intenso, non ho trovato una curva così netta come quella della fig. 8. Perciò l'espressione (5) di $\Delta$, dedotta empiricamente dalla fig. 8 , ha probabilmente bisogno di qualche

1) Phil. Mag., (5.*), 10, p. 304, 1880.

3) Wiener Ber., 94, 1887 a pag. 561. 
lieve ritocco. In ogni caso però $\Delta$ si è presentato come una funzione pari di $H$, e periodica con periodo $\pi$, rispetto $a \varphi$.

A questo proposito, importa stabilire la seguente proposizione che può servire di guida nelle ricerche sperimentali: «Se un fenomeno galvanomagnetico varia col quadrato del campo, ciò̀ se ̀̀ funzione pari di $\mathrm{H}$, esso, considerano come funzione $d i \varphi$ ha periodo $\pi$, e viceversa. Se invece è funzione dispari, cioè si inverte col campo, esso ha il periodo $2 \pi$ e viceversa.

Infatti nella prima ipotesi :

$$
f(\varphi,-\mathrm{H})=f(\varphi, \mathrm{H}) ;
$$

e poichè evidentemente aumentare $\varphi$ di $180^{\circ}$ equivale a invertire il campo, si avrà

quindi :

$$
f\left(\varphi+180^{\circ}, \mathrm{H}\right)=f(\varphi,-\mathrm{H}),
$$

$$
f\left(\varphi+180^{\circ}, \mathrm{H}\right)=f(\varphi, \mathrm{H}) \text {. }
$$

La reciproca è evidente, e lo è pure la seconda parte del teorema. I casi più frequenti in natura sono quelli di semplice proporzionalita, e così nell'effetto studiato abbiamo un fenomeno proporzionale al campo, che si esprime mediante una somma di due funzioni a periodo $2 \pi$ cioè $\operatorname{sen} \varphi \theta \cos \varphi$, ossia si scinde in una componente trasversale $\theta$ in una assiale, $\theta$ si ha poi la perturbazione proporzionale ad $\mathrm{H}^{2}$.

Questa si potrà esprimere in generale con la serie di Fourier

$$
\begin{gathered}
a_{\varphi}+a_{4} \operatorname{sen} \varphi+a_{8} \operatorname{sen} 2 \varphi+a_{3} \operatorname{sen} 3 \varphi+\ldots \ldots \\
+b_{1} \cos \varphi+b_{8} \cos 2 \varphi+b_{3} \cos 3 \varphi+\ldots \ldots
\end{gathered}
$$

nella quale si cancellino i termini dispari : ossia sarà formata tutta di armoniche pari.

Così girando la laminetta, si può separare l'effetto lineare in $\mathrm{H}$, da quello quadratico. In ciò sta appunto l'importauza della proposizione dimostrata, la quale da l' intima ragione della presente ricerca, perchè dice che dal modo col quale varia 
l'effetto rispetto a $\varphi$ si può dedurre come varia rispetto ad. $\mathbf{H}$.

Tornando ora alle formole $(6),(7)$ che in campi deboli si possono considerare come del tutto rigorose, seguiamole più da vicino iu campi forti. Le esperienze mostrano che esse continuano a sussistere con buona approssimazione, purchè si modifichino leggermente le costanti. Insomma queste sono veramente delle variabili lente, come d̀ ad esempio la costante $R$ dell'effetto comune, e ciò avviene perchè negli effetti galvano e termomagnetici, si presenta la nota tendenza alla saturazione; al crescere del campo, gli effetti crescono meno di quanto vorrebbe la proporzionalità ad $\mathrm{H}$, o ad $\mathrm{H}^{2}$.

Che cosa dovremmo aspettarci nelle esperienze presenti, se questo fenomeno non si presentasse, cioè se le quattro costanti rimanessero perfettamente immutate?

$\nabla i$ sono grandezze lineari cioè proporzionali ad $\mathbf{H}$ ed altre quadratiche ciò̀ proporzionali ad $\mathrm{H}^{2}$.

Dovrebbero essere lineari:

la diagonale secondaria del quadrilatero caratteristico,

$$
\Phi=\varphi_{2}-\varphi_{1}=2 \frac{a+b \cos 2 \varphi_{0}}{A} \mathrm{H}
$$

la media $m$ degli effetti che si hanno invertendo campo e corrente primaria cioè la media di due curve dette vicine (coppia $($ ) $\leftrightarrow,(X) \lll$ ovvero $(\cdot) \lll,(X) \ggg)$

$$
m=\mathrm{AH} i \operatorname{sen}\left(\varphi-\varphi_{0}\right) \text {; }
$$

le ordinate dei quattro punti di incontro di due curve vicine che si esprime pure con $m$.

Si possono considerase come quasi lineari le ordinate massime delle curve, perchè ivi il termine in $\mathrm{H}^{2}$ se non è propriamente nullo, è però trascurabile di fronte al termine in $\mathrm{H}$, almeno a giudicare dalla laminetta studiata.

Dovrebbero essere quadratiche:

$$
2 y_{0}=2\left(a+b \cos 2 \varphi_{0}\right) \mathrm{H}^{i} i,
$$


e la differenza

$$
\Delta=2(a+b \cos 2 \varphi) \mathrm{H}^{2} i \text {. }
$$

Alcune di queste grandezze si possono determinare con maggiore, altre con minore precisione, e quindi le misure meritano diversa fiducia, ma tutte vengono direttamente dalla esperienza. Per esempio per determinare $y_{0}$ basta girare la laminetta, finchè per tentativi si riesca a cogliere una posizione nella quale lasciando fissa la corrente primaria e invertendo il campo, la deviazione galvanometrica non cambi nè in valore nè in segno: questa è appunto $y_{0}$ - contemporaneamente ecco determinato $\varphi_{0}$.

Riprendiamo prima la fig. 5 e di là ricaviamo $y_{0}$, e per angoli piccoli anche $m, \Delta$. A quelle esperienze aggiungo anche le seguenti fatte per la posizione fissa $\varphi=45^{\circ}$, cosicchè $m, \Delta$ si hanno anche per questa posizione

$$
\varphi=45^{\circ}
$$

\begin{tabular}{c|c|c|c|cc|c|c}
\hline$H$ & 1600 & 2600 & 3400 & 3900 & 4400 & 5700 & 6300 \\
\hline$(\cdot) *$ & 44 & 71 & 86 & 99 & 108 & 132 & 141 \\
$(X) \%$ & 41 & 66 & 82 & 90 & 93 & 105 & 110
\end{tabular}

Riportando tutti $i$ dati in funzione del campo, si ha la fig. 9, nella quale $\Phi, m(o), m(45)$ dovrebbero essere rette; $y_{0}, \Delta(0), \Delta(45)$ parabole, ossia $\sqrt{y_{0}}, \sqrt{\Delta(0)}, \sqrt{\Delta(45)}$ anche esse rette. Ora invece di rette, si hanno, ad accezione di $m(0)$, curve più o meno concave in giù, quindi gli effetti crescono meno della proporzione con $\mathrm{H}$ o con $\mathrm{H}^{2}$ rispettivamente.

§ 4. - Il comportamento descritto rivela una tendenza più, o meno spiccata verso uno stato di saturazione, e quasi quasi si potrebbe azzardare 1'ipotesi che la suscettività del bismuto non sia del tutto costante. 
Ma anzichè invischiarmi nel campo un po' vago delle congetture, preferisco proceders coi piedi di piombo e mi limito strettamente a riferire $\theta$ commentare i dati delle esperienze.

Il' problema più importante è ora quello di vedere se gli effetti assiale, trasversale e la perturbazione, si avvino tutti verso una saturazione, cioè se si scostino allo stesso modo, o no dalle semplici leggi di proporzionalità : così ci potremo formare un'idea della connessione di questi diversi fenomeni attraverso il campo.

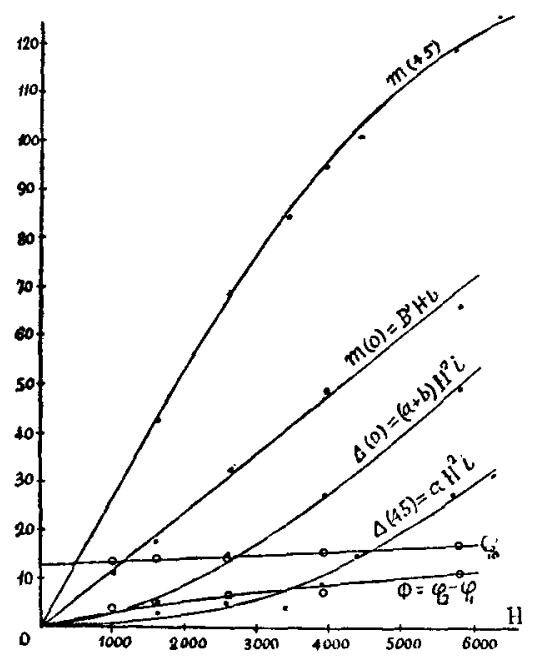

fig. 9

Un tale studio ritengo, debba essere molto utile in vista delle spiegazioni che le diverse teorie sopratutto quelle elettroniche hanno tentato e tentano di dare per i fenomeni galvano e termomagnetici, spiegazioni che, a 3 essere sinceri, son riuscite con scarsa fortuna.

Uno sguardo alla fig. 5 ci mostra intanto che $\varphi_{a}$ non è perfettamente costante, e quindi ricordando che $\operatorname{tang} \varphi_{0}=\frac{B^{\prime}}{A^{\prime}}$, si deve concludere che al crescere del campo muti il rapporto 
fra l'effetto assiale e quello trasversale. Altri indizi sul modo col quale si comportano le costanti al crescere del campo si possono dedurre dalla fig. 9; ma invece di procedère saggiando, poichè le esperienze descritte permettono di separare i diversi effetti, è bene procedere a ciò, determinando le costanti. Ritengo che il modo più semplice sia il seguente:

Poniamo per brevità:

$$
\begin{aligned}
& y_{1}=\text { ordinata di }(\cdot) \ggg \quad y_{3}=-y_{1} \text { ordinata di }(\cdot) \\
& y_{3}=\text { ordinata di }(X) \cdots \quad y_{4}=-y_{2} \text { ordinata di }(X) \\
& \left.\begin{array}{rl}
m=\frac{y_{1}+y_{4}}{2}=-\frac{y_{3}+y_{2}}{2} & =\frac{y_{1}-y_{2}}{2}=\mathrm{AH} i \operatorname{sen}\left(\varphi-\varphi_{0}\right) \\
& =\mathrm{A}^{\prime} \mathrm{H} i \operatorname{sen} \varphi-\mathrm{B}^{\prime} \mathrm{H} i \cos \varphi
\end{array}\right\} \begin{array}{l}
\text { manca } \\
\text { la per- } \\
\text { turbaz. }
\end{array} \\
& \frac{\Delta}{2}=\frac{y_{1}-y_{4}}{2}=\frac{y_{1}+y_{2}}{2}=(a+b \cos 2 \varphi) \mathrm{H}^{2} i \text { sola perturbaz.; }
\end{aligned}
$$

ed osserviamo che si passa da (6) a (7) mediante le formole

$$
\left.\begin{array}{l}
\mathbf{A}^{\prime}=\mathbf{A} \cos \varphi_{0}, \text { massimo effetto trasversale } \\
\mathbf{B}^{\prime}=\mathbf{A} \operatorname{sen} \varphi_{0}, \text { massimo effetto assiale }
\end{array}\right\} \operatorname{tang} \varphi_{0}=\frac{\mathbf{B}^{\prime}}{\mathbf{A}^{\prime}}
$$

cioè $\mathrm{A}^{\prime}, \mathrm{B}^{\prime}, \mathrm{A}$ formano un triangolo rettangolo dove $\mathrm{A}$ è $\mathrm{l}^{\prime}$ ipotenusa e l'angolo $\varphi_{0}$ è opposto al cateto $\mathrm{B}^{\prime}$.

Allora per ricavare le costanti, noto $\varphi_{0}$, basta studiare l'effetto per i valori seguenti di $\varphi$ :

perchè si ha

$$
04590+\varphi
$$

$-m(o): m(90): m\left(90+\varphi_{0}\right)=\mathrm{B}^{\prime}: \mathrm{A}^{\prime}: \mathrm{A}=\operatorname{sen} \varphi_{0}: \cos \varphi_{0}: 1$

$$
\Delta(0): \Delta(45): \Delta(90)=(a+b): a:(a-b) ;
$$

anzi così si hanno dati sovrabbondanti che permettono molteplici controlli fra i risultati.

Non ho tutti i dati per eseguire per disteso questa determinazione completa, nè posso riprendere le esperienze con la stessa lastrina che si è rotta, quindi mi debbo limitare agli elementi riportati nella fig. 4 e nella fig. 9, che in sostanza forniscono $\varphi(0), m(0), \Delta(0), \Delta(45)$. 
1. - Anzitutto voglio ripetere che secondo la fig. 4 con l'annessa tabellina, $\varphi_{0}$ anzichè essere costante, varia un poco e precisamente con legge lineare; cioè dà $12^{\circ}, 5$ per $\mathrm{H}=0$, a $17^{\circ}$ per $\mathrm{H}=6000$.

2. - Poichè, in fig. 9, $m(0)=\frac{y_{1}(o)-y_{2}(0)}{2}=-\mathrm{B}^{\prime} \mathrm{H} i$, è una retta, si conclude che $B^{\prime}$ è costante (nelcaso presente =10), ossia che l'effetto assiale segue csattamenle la proporzionalitù rispetto al campo; e ciò fino ad $\mathrm{H}=6000$.

3. - Per conseguenza, la costante dell'effetto (Hall) trasversale $\mathrm{A}^{\prime}=\frac{\mathrm{B}^{\prime}}{\operatorname{tang} \varphi_{0}}$ non resta fissa al crescere del campo, perchè tang $\varphi_{0}$ cresce, e quindi $A^{\prime}$ decresce lievemente. Tanto è d'accordo con tutte le esperienze conosciute, giacchè $A^{\prime}$ non è altro che la solita $R$ a parte un fattore costante. Nei limiti di campo considerati, $A^{\prime}$ varia da 45 per $\mathrm{H}=0$, a 33 per $\mathrm{H}=6000^{\mathrm{i}}$ ).

4. - Se dalla curva $\Delta(o)=y_{1}(o)+y_{2}(o)=2(a+b) \mathbf{H}^{2} i$ si deduce $\sqrt{\Delta(o)}$, si trova, come si è detto, una curva un po' concava in giù, quindi $(a+b)$ non si mantiene costante, ma diminuisce al crescere del eampo.

5. - Se dalla curva $\Delta(45)=y_{1}(45)+y_{8}(45)=2 a H^{3} i$ si leduce $\sqrt{\Delta(45)}$ si trova sensibilmente una retta, quindi $a$ rimane approssimativamente costante $=\mathbf{0 , 3 6}$.

6. - Per conseguenza, poichè $a+b$ decresce ed $a$ resta costante, $b$ deve decrescere. Per sottrazione da

$$
\Delta(0)-\Delta(45)=2 b \mathrm{H}^{2} i
$$

si trova il suo valore che varia da 0,8 per $H=1000$ a 0,3 per $H=6000$. Su questi valori di $a, b$, però non e'è da fare troppo affidamento poichè sono piccoli e uno sbaglio nelle letture può farli mutare notevolmente. È però accettabile la

') Il segno di $\mathrm{R}$ d negativo, come al solito, il valore piuttosto basso, oirca $=3$. 
conclusione che $b$ decresce: probabilmente, come già si disse, l'espressione della perturbazione avrà bisogno di un lieve ritoceo.

Come controllo, coi valori che utilizzando solo $m(o), \Delta(o)$ $\Delta(45)$ sono stati così dedotti, si possono calcolare per esempio $m(45), y_{0}, \Phi$, e confrontarli con le esperienze riportate, 0 anche con la fig. 6 ( $\mathrm{H}=3400 i=1,15$ ). Si trova un buono accordo, sopratutto se si tiene presente che ogni esperienza ̀̀ fatta una volta e quindi non la luogo compensazione di errori per via di medie.

In conclusione fino a 6000 Gauss, al crescere del campo:

$$
\begin{aligned}
& \mathbf{B}^{\prime}=\mathrm{A} \operatorname{sen} \varphi_{0} \text { resta costante } \\
& \text { a . . . . 》 》 } \\
& b \text {. . . decresce molto } \\
& \varphi_{0} \text {. . . . cresce linearmente }
\end{aligned}
$$

$e$ in conseguenza

$$
\begin{aligned}
& A^{\prime}=\frac{B^{\prime}}{\operatorname{tang} \varphi_{D}}(\text { proporz. ad } R) \text { decresce } \\
& A=\frac{B^{\prime}}{\operatorname{sen} \varphi_{0}} \text { decresce }(\text { poco }) .
\end{aligned}
$$

E notevole che $B^{\prime}$ resta costante, mentre $A^{\prime}$ varia; questo indica che l'effetto assiale è più semplice di quello trasversale studiato comunomente. Se successive prove con altre lastre confermeranno le presenti conclusioni, l'effetto assiale fornira il mezzo più semplice e sicuro per la misura dei campi magnetici a preferenza dell'effetto trasversale e delle variazioni di resistenza.

E dunque un vasto campo che si offre all'indagine ulteriore.

§ 5. - Il soggetto sperimentale delle presenti investigazioni è stato sempre la stessa lastrina di bismuto; ora l'interesse dei fenomeni constatati scemerebbe di molto, se essi fossero peculiari dal campione esaminato, e non si ripresen- 
tassero in altre lamine di bismuto o di varii metalli. Gli esperimenti ancora grezzi notati, o citati mostrano l'esistenza dell'effetto assiale nei diversi campioni di bismuto (o tellurio) esaminati; quindi è presumibile che anche tutto il resto seguar l'andamento rilevato dall'esame della lastrina sottile e riproduca, salvo varianti, le modalita viste. Ma ho voluto assicurarmi meglio, e quindi, modificato opportunamente l'apparecchio della fig. 3 , in modo da ottenere completa libertà di rotazione e un'esatta misura degli angoli, ho esaminato prima una lastra spessa di bismuto, e poi una sottile. Senza entrare in particolare, riporterò sotto i diagrammi relativi.

Scelsi prima la lastra grossa (spessore $2,3 \mathrm{~mm}$.) sopratutto per evitare il sospetto che il campo potesse deformare le lastrine metalliche sottili. Riconobbi poi che il fenomeno era più complicato con tale lastra grossa, perchè il galvanometro dava prima una impulsione pronta e poi lentamente andava assumendo una posizione finale diversa. Si tratta certo di fenomeni termomagnetici lenti che si sovrappongono a quelli galvanomagnetici rapidi.

E nota la distinzione che si fa fra effetto Hall isotermico e adiabatico. In poche parole si tratta di ciò: l'effetto Peltier ai contatti fra la lastrina e gli elettrodi primarii, e gli effetti Joule e Thomson nella lastrina producono, o trasportano calore e si stabilisce un certo regime galvanico e termico. Eccitato il campo, viene turbato sia il regime galvanico che quello termico coi noti effetti, che si intralciauo.

Per studiare i fenomeni galvanomagnetici soli si può procedere in due modi: o si deducono dai dati globali delle esperienze, o si stabiliscono condizioni sperimentali forzate, come isotermia, o adiabaticità. $\mathrm{E}$ su questo argomento esistono pregevoli lavori sia teorici che sperimentali. Ora nel caso di una lastrina sottile, si può pensare che essa, appunto per la sua sottigliezza, si metta prontamente in equilibrio termico con l'ambiente; quindi l'effetto termico sparisce del tutto, o si nasconde, tanto più che avendosi con lastre sottili effetti Hall 
più cospicui, si pnò lnvorare con deboli correnti primnrie, mentre con lastre relativamente grosse si devono adoperare correnti più forti.

Tenendo presente che l'effetto galvanico è rapido, mentro quello termico è lento, si potrà forse ricavare un metodo spicciativo per separare i due effetti nelle lamine spesse, seguendo accuratamente il galvanometro, purchè l'equipaggio abbia un piccolo periodo di oscillazione. Ma intanto credetti più prudente tornare, per 10 meno in un primo tempo, a lastre più sottili e ne costruii una dello spessore di $0,37 \mathrm{~mm}$.

Ecco ora, i diagrammi ottenuti con le due lastre. Per quella più grossa, avendo tolto la resistenza in serie col galvanometro, allo scopo-di aumèntare la sensibilità, $1 \mathrm{~mm} . \equiv 0,6$ microvolt.

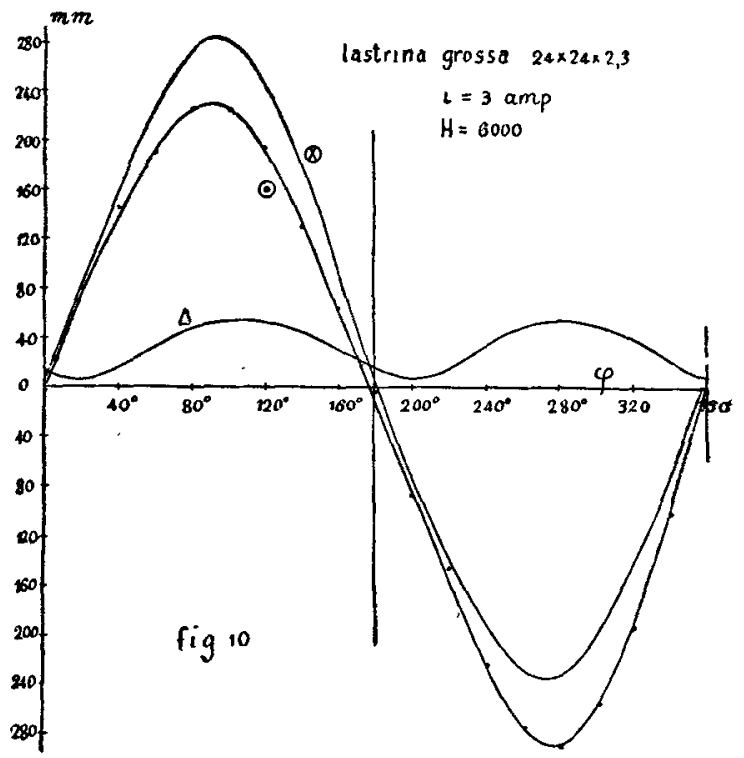

Da essi si rileva:

Nel caso a) si presenta oltre all'effetto trasversale anche un notevole effetto perturbatore. Invece manca del tutto, o quasi, l'effetto assiale giacchè $\varphi_{0}$ è quasi nullo. 
Nel casa b) vi sono tutti e tre gli effetti e, cosa notevole, quello assiale è molto più spiceato del solito in modo che l'effetto massimo si ha per una posizione notevolmente diversa dalla verticale $(\varphi= \pm 90)$ cioè da quella nella quale l'effetto Hall ̀̀ abitualmente studiato.

La perturbazione questa volta può essere rappresentata approssimativamente dalla formola

$$
\frac{\Delta}{2}=6-6 \cos 2 \varphi,
$$

che è dello stesso tipo di quella trovata per la lastrina del $\S 2$ ma con la costante b negativa.

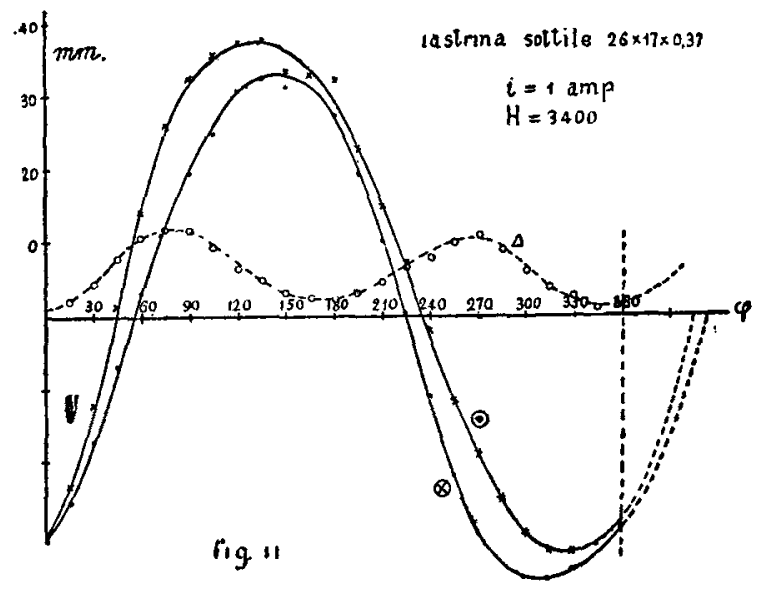

Da che cosa possano dipendere queste particolarità è difficile dire. Certo ha importanza grandissima in queste ricerche lo stato cristallino della sostanza e il suo grado di purezza; ed è pure assodato che le proprietà fisiche di un metallo dipendono dal processo termico, col quale si è ottenuto, ossia dalla sua storia termica "). Constatato così che i tre effetti trasversale assiale, pertubatore non sono peculiarità della la-

1) E. Gohen e G. De Bruin. - Die Metastabilität d. Metalle ecc. Zeitschr. f. phys. Chem., 89, pag. 748, 1915. 
strina esperimentata da principio, ma sussistono in misura più, o meno grande in altri casi, si può concludere che in generale, per lo meno col bismuto, $i$ tre effetti sussisteranno insieme $\theta$ si offre, anzi si impone, uno studio sistematico di essi.

Questo studio si agevola modificando, come ho fatto, l'apparecchio della fig. 3 , in modo che consenta libertà di rotazione e permetta di cambiare la lastrina in modo comodo.

Voglio aggiungere che anche con questa seconda lastrina sottile, variando l'eccitazione del campo, ho ritrovato fra l'altro che $\varphi_{0}$ varia leggermente e linearmente; e che $\mathrm{B}^{\prime}$ resta costante cioè che l'effetto assiale si mantiene proporzionale al campo. Così almeno fino ad $\mathrm{H}=6000$.

§6. - E a questo punto dovrei, prima di chiudere il lavoro, tentare una spiegazione teorica dei fatti studiati. A ciò jo rinunzio per ora, non tanto per seguire l'eminente precetto «Hypoteses non fingo 》 a dir vero attualmente un po' in disuso, quanto perchè il materiale delle esperienze mi sembra ancora insufficiente. Non nego che combinando i principali fenomeni galvanomagnetici coi principali termomagnetici ${ }^{1}$ ) si riesca ad architettare una spiegazione, ma è difficile dire quale grado di probabilità le si possa attribuire.

Qui cade in acconcio una considerazione di carattere generale. Se si conosce già un certo gruppo $\mathbf{A}, \mathbf{B}, \mathbf{C}, \ldots \mathrm{D}$, di denomeni e se ne riesce a spiegare un altro $\mathrm{X}$ mediante quelli, si suol dire che $X$ è un fenomeno secondario, o un effetto, e A, B, C.... D sono primari o cause. Ma molte volte queste distinzioni sono arbitrarie e dipendono dal caso, che ha fatto trovare $\mathrm{X}$ dopo legli altri, e dalla comodità; e forse si potrebbe mediante il gruppo $\mathrm{X}, \mathrm{B}, \mathrm{C} ; \ldots . . \mathrm{D}$ spiegare $\mathrm{A}$, che diventa cosl secondario. La distinzione non ha vero fontamento logice se non quando o si trovino le cause remote, o

7) Se ne contano almeno 10, (Vedasi nel Winkelmann, pag. 459) oltro I'effetto Hall. 
si riesca a fare uno schema simile a quelli che usa l'analisi per decidere per esempio di un dato sistema di variabili quante si possano considerare come indipendenti e quante come funzioni, osservando i reciproci legami già stabiliti. Quindi, se anche si assodasse che l'effetto assiale e quello perturbatore si spiegano mediante gli altri fenomeni conosciuti, i due nuovi non perderebbero il loro interesse, e anzi viceversa sarebbe da tentare se mediante essi non si spieghi qualche fatto noto prima. Importa inoltre tener presente che oltre alle ragioni di necessità logica ve ne possono essere di comodità o semplicità logica, analoghe a quelle per cui in analisi si sceglie come variabile l'arco e non il seno.

Quest' ultima osservazione potrebbe forse essere invocata in favore dell'effetto assiale, se - come sembra - esso è veramente più semplice di quello trasversale.

Ad ogni modo simili speculazioni, o sopratutto la trattazione matematica, non potranno arere solido fondamento, se non quando si siano ripetute le esperienze qualitative e quantitative descritte con campioni di bismuto o altri metalli perfettamente determinati - intendo in primo luogo chimicamente; e cristallograficamente.

Voglio soltanto concedermi la libertà di richiamare da una parte alcuni dei fatti che possono avere maggiore attinenza con quelli studiati qui, e di gettare d'altra parte uno sguardo fuggitivo alle teorie più importanti.

Ricordiamo prima di tutto che la perturbazione è un effetto quadratico in senso largo, cioè dipende (linearmente o no) dal quadrato del campo. Ora i soli effetti quadratici fin qui conosciuti sono quelli longitudinali uno termico e l'altro galvanico, il quale ultimo si interpreta - o forse si fonde - con una variazione di resistenza. Conformemente al teorema del $\$ 3$ questo effetto, che è funzione pari di $H$, deve essere funzione periodica a periodo $\tau$ dell'angolo che il piano della spiralina di resistenza forma col campo e infatti Lenard ') ha

1) Wied $A n n .39,1890$, p. 90. 
trovato per la resistenza nel campo magnetico l'espressione

$$
\mathrm{W}=a+b \operatorname{sen}^{2} \theta-C \operatorname{sen}^{2} \theta \cos 2 \theta,
$$

e C. Carpini ') quella più semplice

$$
\mathrm{W}=\mathrm{W}_{0}+\mathrm{A}(1-\cos 2 \alpha),
$$

l'una e l'altra a periodo $\pi$.

In quanto all'effetto termomagnetico longitudinale, esso come è noto, si riduce a questo: prodotta una differenza di temperatura nei soliti attacchi primari A,B di una lastrina e compensata la f. e. m. termoelettrica, si ripresenta una corrente nel circuito primario quando si eccita il campo. Questo fatto probabilmente è dovuto - o si sovrappone - alla vaviazione del potere termoelettrico ai contatti rame-lastrina in A e B, nel qual caso il fenomeno si dovrebbe metter d'accordo con le esperienze e la teoria del prof. La Rosa sulla variazione del potere termoelettrico in un campo magnetico \%). E come l'effetto termomagnetico longitudinale è quadratico, lo stesso deve avvenire per la variazione dell' effetto Peltier, ciò̀ esso deve essere indipendente dal verso del campo - cosa d'altronde ovvia. Allora c'è da aspettarsi che l'effetto Peltier nel campo varii al variare dell'orientazione, con legge periodica a periodo $\pi$, e sarebbe interessante una conferma sperimentale.

Ora si può pensare che la perturbazione, come effetto quadratiço si spieghi con uno degli altri effetti. Non escludo ciò, ma non ritengo che la dipendenza sia immediata. Sarebbe errato per esempio dire che la lettura dell'effetto Hall muti invertendo il campo unicamente perehè muta la resistenza fra gli attacchi secondari.

Infatti la resistenza interna della lastrina fra questi punti $-0,3 \Omega$ circa - e trascurabile di fronte alla resistenza esterna di $130 \Omega$, e quindi la d. d. p. coincide cou la f. e. m.,

') N. Cim., 8, 1904, p. 171.

2) N. Cim., 18, p. 26, 1919 ; ibidem, p. 39. 
la quale è quella che è, indipendentemente dalla resistenza. Il legame quindi fra $\mathrm{i}$ due fenomeni è certo più recondito.

A mio parere, è più logico pensare che, siccome i quattro effetti notati, cioè perturbazione, variazione di resistenza, effetto termomagnetico longitudinale, variazione di effetto Peltier sono tutti quadratici, essi debbano ricondursi a cause comuni, delle quali sarebbero manifestazioni diverse. E queste cause potrebbero essere quelle stesse stabilite dal La Rosa per spiegare il secondo e il quarto degli effetti ora ricordati, cioè: il numero degli elettroni liberi all'interno del metallo muta sotto l'azione del campo e il cammino medio libero si incurva e si allunga. Se poi si fa girare nel campo il soggetto sperimentale supposto isotropo, si potrà spiegare come il fenomeno varii, utilizzando una soła componente di H. Ma. poichè, secondo eleganti esperienze di Van Everdingen ') confermate da altri, la variazione di resistenza di nna sbarra di bismuto cristallino (sistema esagonale) è diversa secondo che la sbarra (disposta normalmente al campo) è tagliata lungo una; o un'altra direzione dal cristallo originario, la teoria per spiegare questo non potrà fare a meno di entrare nella considerazione profonda della struttura cristallina, abbandonando il comodo cammino medio libero, che può reggere solo per i corpi isotropi.

Lo stesso Van Everdingen ha sperimentato i suoi prismi di bismuto cristallino per rispetto all'effetto Hall, ed è venuto alla importante conclusione che esso varia nei diversi campioni, in modo che rappresentando $\frac{1}{\sqrt{R}}$ con un vettore, orientato rispetto a una retta fissa come sono le linee di forza rispetto all'asse cristallino, si ottiene un ellissoide di rotazione. Nelle sue esperienze si trattava dell'effetto Hall comune $(\varphi=90)$ e quindi manca la componente che ho chiamato assiale, e siccome per togliere la dissimmetria si suol fare, come fu avvertito

1) Se ne ha un buon sunto fatto dall'autore stesso, nell'Areh. d. Sc. phys et nat., (4), 11, 1901, pag. 433. Qualche cosa d detta nel Winkelmann. 
al $\S 2$, la media fra $(X),(\cdot)$ manca forse anche la perturbazione. 'Tuttavia si intuisce che debba esserci uno stretto nesso fra le esperienze sul tipo di Everdingen e quelle sul tipo da me descritto, giacchè il fenomeno complessivo in una lastrina non isotropa girevele deve dipendere sia dall'orientazione di essa rispetto al campo, sia dall'orientazione dell'asse cristallino rispetto alla lamina.

Vediamo ora in quale delle teorie principali i fenomeni esposti e in particolare quello assiale si possano con più pro. babilità inquadrare. Esistono da un canto le teorie antiche, che si possono dire anche formali o esteriori, di fronte alle teorie elettroniche, moderne, che cercano di entrare nell'intimo del corpo.

Le teorie antiche, che fanno capo a Lorentz e a Boltzmanu sono prive di modello: esse prendono le espressioni

$$
\frac{\partial \varphi}{\partial x}=-\frac{1}{\mathrm{~K}} i_{x}, \quad \frac{\partial \varphi}{\partial y}=-\frac{1}{\bar{K}} i_{y}, \quad \frac{\partial \varphi}{\partial z}=-\frac{1}{\mathbf{K}} i_{z},
$$

che in sostanza sono la traduzione della legge di Ohm e danno il movimento dell'elettricità funri del campo magnetico, e le modificano opportunamente, in modo che integrando le equazioni, vi sia coincidenza coi fenomeni osservati.

Queste teorie se non ci fanno osservare la vita dell'elettrone, o dell'atomo, hanno però il pregio di una grande generalità, per la quale si possono facilmente adattare ai diversi fenomeni. $\mathbf{E}$ appunto rivangando nel passato trovo un pregevole articolo di Goldhammer ') nel quale è trattato il caso, fra gli altri, di una laminetta parallela al campo.

Quest'autore modifica anche lui le equazioni suddette, ma fa un passo avanti perchè interpreta queste modificazioni ammettendo che un corpo isotropo introdotto nel campo perda l' isotropia e così spiega bene l'effetto Hall. Trattando in tal modo il caso di una lastra parallela al campo e percorsa da

') Wied Ann., 31, 1887, pag. 370 . 
corrente normale ad esso, le equazioni integrate danno da un canto una f. e. m. normale al campo, cioè il solito effetto Hall, qucsta volta nel senso dello spessore della lamina e con eguale ') costante $R$, e dall'altro canto una f. e.m. lungo il campo, cioè l'effetto che ho chiamato assiale. Se non che, dalla discussione che fa poi il Goldhammer si conclude implicitamente che l'effetto assiale deve sparire. Ciò è nettamente contraddetto dalle presenti esperienze; ma veramente esse contraddicono non tanto le equazioni di Goldhammer, quanto le deduzioni che egli ne fa, fondandosi su considerazioni attinenti a simmetria. Può darsi che queste non abbiano validità nel caso presente, tanto più che l'ipotesi di partenza che il corpo sia originariamente isotropo non è applicabile al bismuto che cristallizza nel sistema esagonale - quindi probabilmente modificando un poco la teoria di Goldhammer si riescirà a spiegare per questa via l'effetto assiale. Resterebbe poi sempre a spiegare la perturbazione.

Passiamo alle teorie elettroniche: esse cercano di spiegare il meccanismo delle intime particelle, che sono il Deus ex machina dei fenomeni, e in molta parte vi riescono senza dubbio. Ma la teoria unitaria, così come essa è ora, ha già molto da fare per piegarsi, o torcersi, in modo da spiegare il diverso segno che l'effetto Hall trasversale ha nei diversi metalli, e quindi è da ritenerc che meno che mai potrà prestarsi a spiegare quello assiale. La stessa difficoltà però si presenterà anche per la teoria dualistica. Essa spiega, è vero, il doppio segno, e in ciò nessuna sorpresa, pechè invece di un solo parametro $\mathrm{N}=$ numero di elettroni negativi liberi per cm. ${ }^{3}$ ne ha due $\mathrm{N}_{1}, \mathrm{~N}_{2}$, anzi invece di un gruppo $(N, l, v)$ ne ha due: ma anch'essa come potrà spiegare un effetto assiale?

Come potrà spiegare il fatto - messo fuori dubbio dalle presenti esperienze - che in una lamina parallela alle linee di forza si manifesti una f. e. m. lungo il campo quando le azioni 
elettrodinamiche, sulle quali le teorie elettroniche fondano la spiegazione dell'effetto Ball, avvengono in un piano normale al campo?

Secondo il mio modesto avviso, anche qui non si potrà avere un successo reale, se non si assegnerà una parte principale del fenomeno alla struttura cristallina ") e cosi forse la stessa teoria unitaria che è preferibile alla dualistica - se non altro perchè đ̀ più semplice - potrà spiegare sia il doppio segno, sia l'effetto assiale. Insomma la spiegazione dei fenomeni si potrd avere solo quando si saprà come rimbalzano gli elettroni dopo un urto proprio o improprio, perchè allora se gli ostacoli girano - o si comportano come se girassero - muta jl regime; gli effetti galvanomagnetici saranno dati dalla rotazione degli ostacoli, cioè dall'azione del campo sui complessi corpuscolari vincolati, combinata con l'azione del campo süi corpuscoli liberi.

La considerazione - in prima linea - della struttura cri stallina si impone anche in vista del fatto che non solo nel mercurio $o$ in altri liquidi ma anche nello stesso bismuto li quefatto, $o$ in quello ottenuto elettroliticamente, secondo vecchie e nnove esperienze ') il fenomeno Hall o manca del tutto, o è così piccolo da potersi facilmente imputare o a cause di errore o, nel caso di liquidi, ad azioni ponderometrici. Ma anche dalle mie esperienze ricavo un conforto a questo modo di vedere, dalla circostanza che in una lastra (fig. 11) l'effet to assiale è cresciuto a seapito di quello traśversale; ciò mi fa pensare grossolanamente a un flusso d'acqua che spinto in un senso si riversa di qua o di là, secondo l'ostacolo contro cui urta e la via che trova libera.

1) Mi sembra che qualche tentativo - a dir vero, finora infruttuoso - sia stato gia fatto in questo senso da P. Gruner, come rilevasi da un articolo di J. Koenigsberger e G. Gottstein - Phys. Zeit., 14, p. 232, 1913.

2) In particolare: Amerio. N. Cimento, 1, p. 342, 1901. Ultimamente se ne deccupato anchs T. Colloui, N. Cimento, 19, p. 163, 1920. 
E voglio pernettermi nua congettura: se i rimbalzi contro gli ostacoli hanno parte importante nêi fenomeni, alla superficie del corpo questi si presenteranno in modo diverso che non allo interno del corpo, dove per il disordine perfetto o imperfetto le azioni si compensano. Quindi, se penetrassimo in un corpo, dovremmo forse aspettarci effetti galvanomagnetici diversi che alla superficie: come pure diversi sarebbero se la lastra invece di trovarsi nell'aria, fosse immersa in un mezzo di proprietà $\theta$ in ispecie di permeabilità differenti.

$\mathrm{Si}$ potrebbero tentare adeguate esperienze per avvalcrare o rigettare tale congettura. Ma del resto, la semplice constatazione che con le considerazioni dell'anisotropia prodotta dal campo nel metallo la vecchia teoria di Goldhammer spiega comodamente l'effetto Hall trasversale e quasi quasi quello assiale, $\mathrm{e}$ il fatto che questa ipotesi dell'anisotropia è confortata dalle esperienze posteriori di Everdingen sulle variazioni di resistenza del bismuto cristallino, deve spingere la teoria elettronica a mettersi risolutamente per questa via.

Non $\nabla^{\prime}$ ha dubbio che se nel calcolo si introducono le.considerazioni strutturali, da un lato possono eventualmente sorgere i dubbi sulla legittimita delle ipotesi - dubbi che occorrerd dileguare con prove sperimentali - dall'altro la teoria elettronica perde il pregio della semplicità meravigliosa che consiste nella sintesi di molti fenomeni disparati, i quali vengono ridotti alla conoscenza di tre soli parametri $\mathrm{N}, l, v$. Ma un giorno, o l'altro, questo sforzo si dovrà fare, non tanto, a dir vero, per illustrare i fenomeni che sono ormai noti, quanto per giustificare la teoria stessa la quale ha la pretesa di pre. sentare un modello di tutti i fenomeni.

E forse la considerazione della struttura permetterà alla teoria unitaria di spiegare, oltre al doppio segno dell'effetto Hall, altri fatti, come la deviazione dalla nota legge di Wiedemann e Franz ecc. 


\section{Sommario.}

Si ̀̀ studiato l'effetto galvanomagnetico complessivo in varie lastrine di bismuto, girevoli attorno ad un asse.

Si è trovato che il fenomeno in prima approssimazione è rappresentato da una sinusoide, i cui massimi e minimi non coincidono con la posizione della lastrina normale al campo.

Lo sfasamento $\varphi_{0}$ della sinusoide dà modo di separare due effetti distinti proporzionali al campo e denominati l'uno assiale cioè manifestantesi come una f. e. m. lungo le linee di forza, e l'altro trasversale o effetto Hall puro. In seconda approssimazione, si trova che la sinusoide è deformata da un terzo effetto detto perturbatore che dipende dal quadrato del campo. Questo è la cansa della nota dissimmetria dell'effetto Hall rispetto al campo.

La perturbazione è rispetto all'angolo $\varphi$ una funzione a periodo $\pi$, ossia un armonica pari. Si è dimostrato che ogni effetto dipendente da $\mathrm{H}^{2}$ deve essere composto esclusivamente di armoniche pari, e viceversa.

Si è constatato che al variare del campo fino a un certo limite l'effetto assiale gli si mantiene proporzionale, mentre l'effetto trasversale mostra presto la solita tendenza alla saturazione. Anche la perturbazione mostra nel suo complesso una tale tendenza, mentre una parte di essa segue la proporzionalità con $\mathbf{H}$.

Si è scritta l'equazione generale del fenomeno, che vale si può dire esattamente per campi non molto intensi e a ogni modo rende conto qualitativamente di tutte le particolarità dei fatti osservati.

Fra gli altri diagrammi sperimentali, se ne è trovato uno detto «quadrilatero caratteristico » che forma il nocciolo della questione, perchè una volta stabilito, permette di conoscere tutto l'andamento del fenomeno.

Si è assodato che per definire l'effetto Hall non basta una sola costante $R$, ma ce ne vogliono almeno tre. 
Infine si sono ricordate alcune esperienze e teorie che possono avere più diretta attinenza con i fenomeni studiati.

4 luglio 1920. 